\title{
Water-Quality Data from Lakes and Streams in the Grand Portage Reservation, Minnesota, 1997-98
}

by Thomas $A$. Winterstein

Open-File Report 00-364

Prepared in cooperation with the Grand Portage Reservation 


\section{U.S. Department of the Interior}

Bruce Babbitt, Secretary

\section{U.S. Geological Survey}

Charles G. Groat, Director

Use of trade, product, industry, or firm names in this report is for descriptive or location purposes only and does not constitute endorsement of products by the U.S. Government.

Mounds View, Minnesota, 1999

For additional information write to:

District Chief

U.S. Geological Survey, WRD

2280 Woodale Drive

Mounds View MN 55112

Copies of this report can be purchased from:

U.S. Geological Survey

Branch of Information Services

Box 25286

Federal Center

Denver CO 80225

For more information on the USGS in Minnesota, you may connect to the Minnesota District home page at http://mn.water.usgs.gov

For more information on all USGS reports and products (including maps, images, and computerized data), call 1-888-ASK-USGS

Open-File Report 00-364 


\section{Contents}

Introduction 1

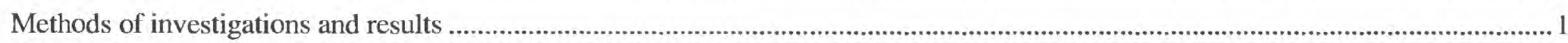

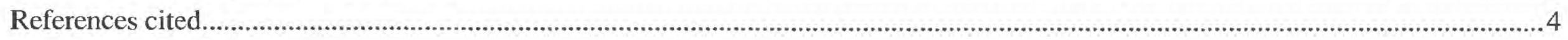

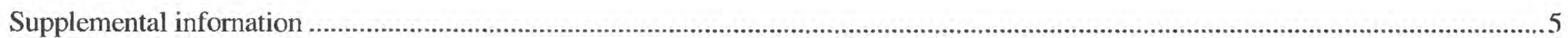

\section{Illustrations}

Figure 1. Map showing location of study area and data collection sites in the Grand Portage Reservation, northeastern Minnesota....... 2

Figure 2. Piper diagram showing percentage distribution of major ion concentrations determined for water sampled from lakes and streams in the Grand Portage Reservation, Minnesota, 1997-98

\section{Tables}

1. Water-quality data collected from lakes and streams in the Grand Portage Reservation, 1997-98

2. Physical characteristics of Cuffs, Little, Loon, and Speckled Trout Lakes, Grand Portage Reservation, 1997-98

3. Relative percent difference between concentrations of constituents in the sample and replicate sample from Speckled Trout and Little Lake

4. Trace metal and organic compounds in the sediments of Cuffs and Loon Lakes, May and September 1998

\section{Conversion Factors, Water-Quality Abbreviations, and Sea Level Datum.}

Multiply

inch (in.)

foot ( $\mathrm{ft}$ )

mile (mi)

degrees Fahrenheit
By

0.3048

1.609

${ }^{\circ} \mathrm{C}=\left({ }^{\circ} \mathrm{F}-32\right) / 1.8$
To obtain

centimeter

meter

kilometer degrees Celsius

Chemical concentrations are given in metric units. Chemical concentrations of substances in water are given in milligrams per liter $(\mathrm{mg} / \mathrm{L})$, nanograms per liter $(\mathrm{ng} / \mathrm{L})$, or micrograms per liter $(\mu \mathrm{g} / \mathrm{L})$. Milligrams per liter is a unit expressing the concentration of chemical constituents in solution as mass (milligrams) of solute per unit volume (liter) of water.

Sea level: In this report, "sea level" refers to the National Geodetic Vertical Datum of 1929 (NGVD of 1929)-a geodetic datum derived from a general adjustment of the first-order level nets of both the United States and Canada, formerly called Sea Level Datum of 1929.

Water year: The water year is October 1 through September 30 and is named for the calendar year in which it ends. 


\title{
Streams in the Grand Portage Reservation, Minnesota, 1997-98
}

\author{
Thomas A. Winterstein
}

\section{INTRODUCTION}

The Grand Portage Reservation is located in northeastern Cook County, Minnesota (fig. 1). In 1997 and 1998 the U.S. Geological Survey (USGS) conducted a study, in cooperation with the Grand Portage Band of Chippewa, to determine the quality of water in selected inland lakes and streams in the Reservation. The USGS collected and analyzed water from two streams, two wetlands, and four lakes (fig 1). Water samples were collected in the spring and fall of 1997 and 1998. In addition, samples of bottom sediments were collected from two of the four lakes in 1998.

The purpose of this report is to present the data collected by the USGS from the study during 1997-98. Water-quality data include temperature, $\mathrm{pH}$, specific conductance, dissolved oxygen, alkalinity, and concentrations of major ions, nutrients, and trace metals. Lake sediment data include concentrations of trace metals and selected organic compounds.

\section{METHODS OF INVESTIGATION AND RESULTS}

Water samples for water-quality analysis were collected from the two streams, two wetlands, and four lakes during June and October 1997, and May and September 1998 (fig. 1, and table 1, at the back of the report). The samples were collected, treated, and stored in accordance with procedures described in Horowitz and others (1994).
The water-quality samples from the four lakes and Swamp Lake Wetland were collected with a Kemmerer sampler. Each of the four lakes were divided into three equal areas and a composite sample was made from equal volumes of water collected at the center of each area. The samples were collected at mid-depth because these lakes are shallow. The sample from Swamp Lake Wetland was collected at mid-depth from the center of a stream that flowed from the wetland into Swamp Lake. The collection point was inside the wetland about one-quarter of a mile upstream from the mouth of the stream. The waterquality samples from Red Rock Creek, Red Rock Creek tributary, and the outlet stream from Center Lake Creek Wetland were collected with a peristaltic pump.

The samples were analyzed at the USGS National Water Quality Laboratory (NWQL) in Arvada, Colorado for major ions, nutrients, and trace metals.

Field measurements (temperature, $\mathrm{pH}$, specific conductance, and dissolved oxygen) were made in accordance with procedures described in Wilde and Radtke (1998). The measurements were made with a portable, multiparameter meter calibrated at the start of each sampling day. In lakes, measurements were made at intervals between the lake surface and lake bottom. Alkalinity was determined by incremental titration.

The field measurements and the analytical results from NWQL are in tables 1 and 2, at the back of the report and in figure 2 .
Six quality assurance samples were collected. Two replicates were collected: one from Speckled Trout Lake during May 1998 and one from Little Lake during September 1998. Four field blanks were collected: one before sampling Red Rock Creek above Sawmill during June 1997, one before sampling Little Lake during October 1997, one before sampling Cuffs Lake during May 1998, and one before sampling Loon Lake during September 1998 (table 1).

Replicate samples are collected to assess the quality, reliability, and precision (reproducibility) of the data generated by the analysis of the samples for chemical constituents. The relative percent difference between the values for the sample and the replicate sample are shown in table 3 , at the back of the report. The relative percent difference was calculated as

$$
\frac{X_{1}-X_{2}}{X_{1}+X_{2}} \times 100
$$

where $X_{1}$ is the value for the sample and $\mathrm{X}_{2}$ is the value for the replicate sample.

Field blanks were collected by passing inorganic-free, de-ionized water through all sample equipment contacted by the actual sample. Field blanks are collected to ensure that equipment cleaning between sampling sites remove all contamination from the previous site, that sampling and sample-processing procedures do not contaminate the equipment, and that transporting and handling the sampling equipment between sites does not contaminate the equipment.

The concentrations of analyzed constituents in the field blank were below the detection limit or very low 


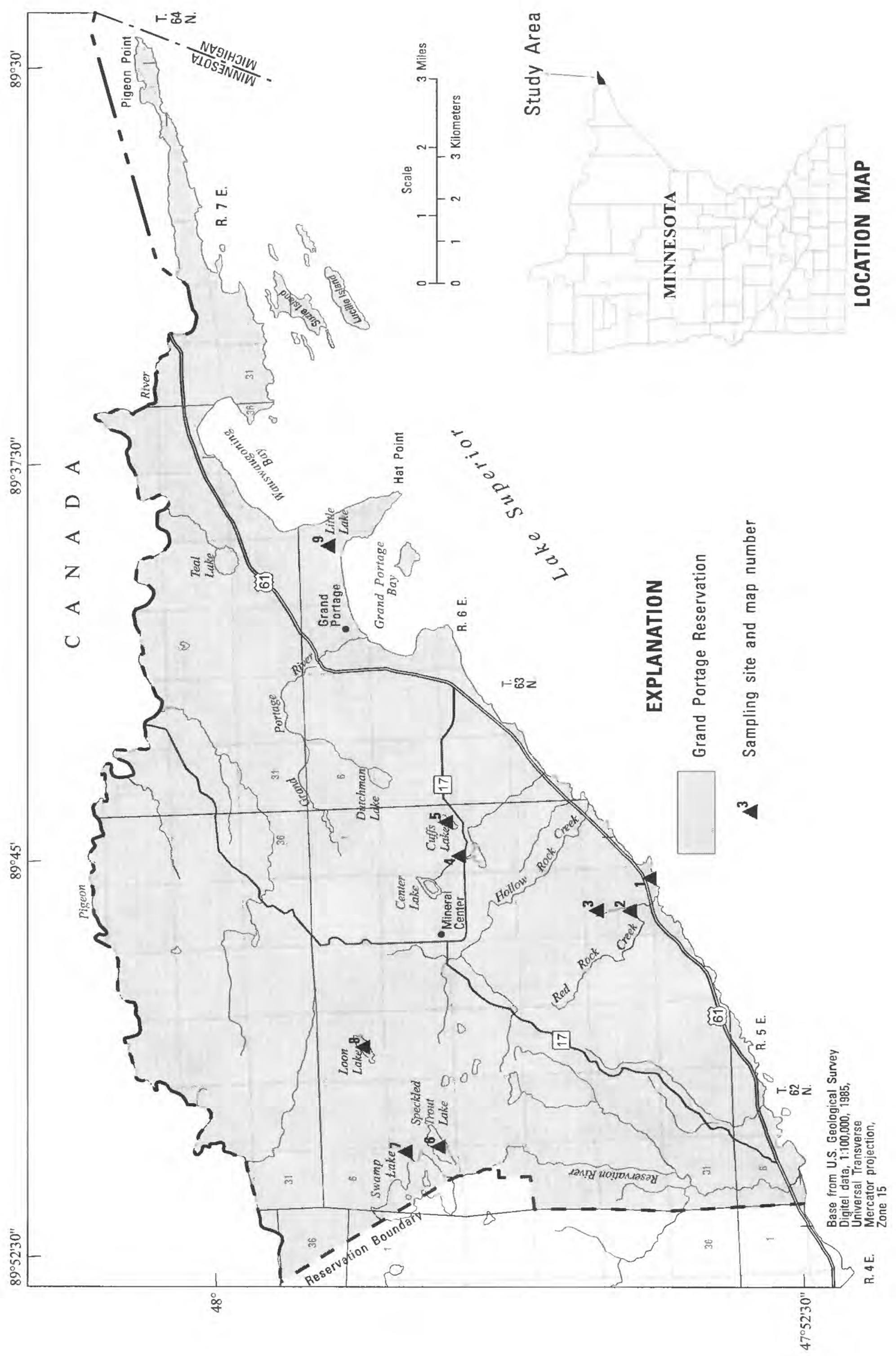

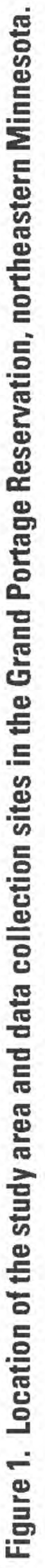




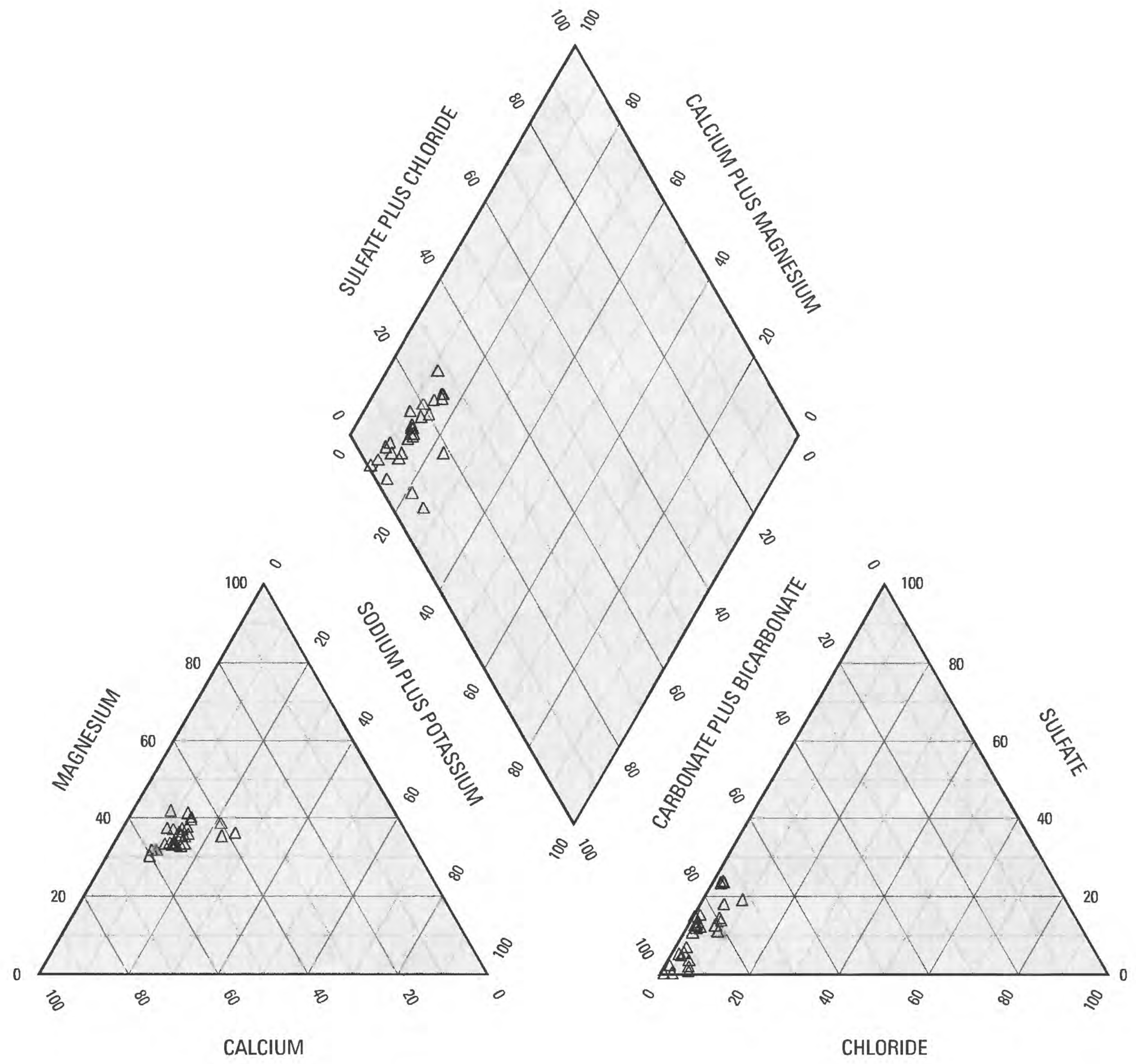

PERCENT DF TOTAL MILLIEQUIVALENTS PER LITER

Figure 2. Percentage distribution of major ion concentrations determined for water sampled from lakes and streams in the Grand Portage Reservation, Minnesota, 1997-98 
compared to the concentrations of the constituents in the samples from the wells, except as noted below. The concentrations of ammonia in the field blanks for Cuffs Lake, May 7 , 1998, and Loon Lake, September 9, 1998, were close to or above the concentrations reported for the samples from the lakes for these days. The concentration of orthophosphate in the field blank for Loon Lake, September 9,1998 , was very close to the concentration reported for the lake for this date. The concentrations of chloride and sulfate were much greater in the field blank for Red Rock Creek above Sawmill, May 5, 1998, than in the sample taken from the Creek that date. The concentration of dissolved silica in the field blank for Little Lake, October 8, 1997, was close to the concentration in the sample taken from Little Lake that day. The concentration of dissolved copper in the field blank for Cuffs Lake, May 7 , 1998 , was close to the concentration in the sample from the lake that day. The concentrations of dissolved cop- per in the field blanks for Loon Lake, September 9, 1998, and Little Lake, October 8, 1997, were as great as concentrations measured in samples from the other lakes and streams.

Lake sediment samples were collected at the center of Cuffs and Loon Lakes. During May 1998, a sample was collected from Loon Lake with a 6-in. by 6-in. by 6-in. Eckman dredge. A polyethylene paddle was used to remove the sample from the sediment collected with the dredge. The sample was removed from the center ot the dredge and about $2 \mathrm{in}$. below the top. During September 1998, samples were collected from Cuffs and Loon Lake with a core barrel made from 2in. acrylic tubing. The samples were taken from the core barrel just below the water-sediment interface. In both lakes the lake sediment is clay- and silt-sized, black, organic material. The bottom material was analyzed for trace metals and selected organic compounds at the Quanterra Incorporated, Arvada, Colorado. The results of the analysis are in table 4 , at the back of the report.

\section{REFERENCES CITED}

Horowitz, A.J., Demas, C.R., Fitzgerald, K.K., Miller, T.L., and Rickert, D.A., 1994, U.S. Geological Survey protocol for the collection and processing of surface-water samples for the subsequent determination of inorganic constituents in filtered water: U.S. Geological Survey Open-File Report 94-539, $57 \mathrm{p}$.

Wilde, F.D., and Radtke, D.B., eds., 1998, Field measurements: U.S. Geological Survey Handbooks for WaterResources Investigations, Book 9, Chapter A6, unpaged. 
SUPPLEMENTAL INFORMATION 


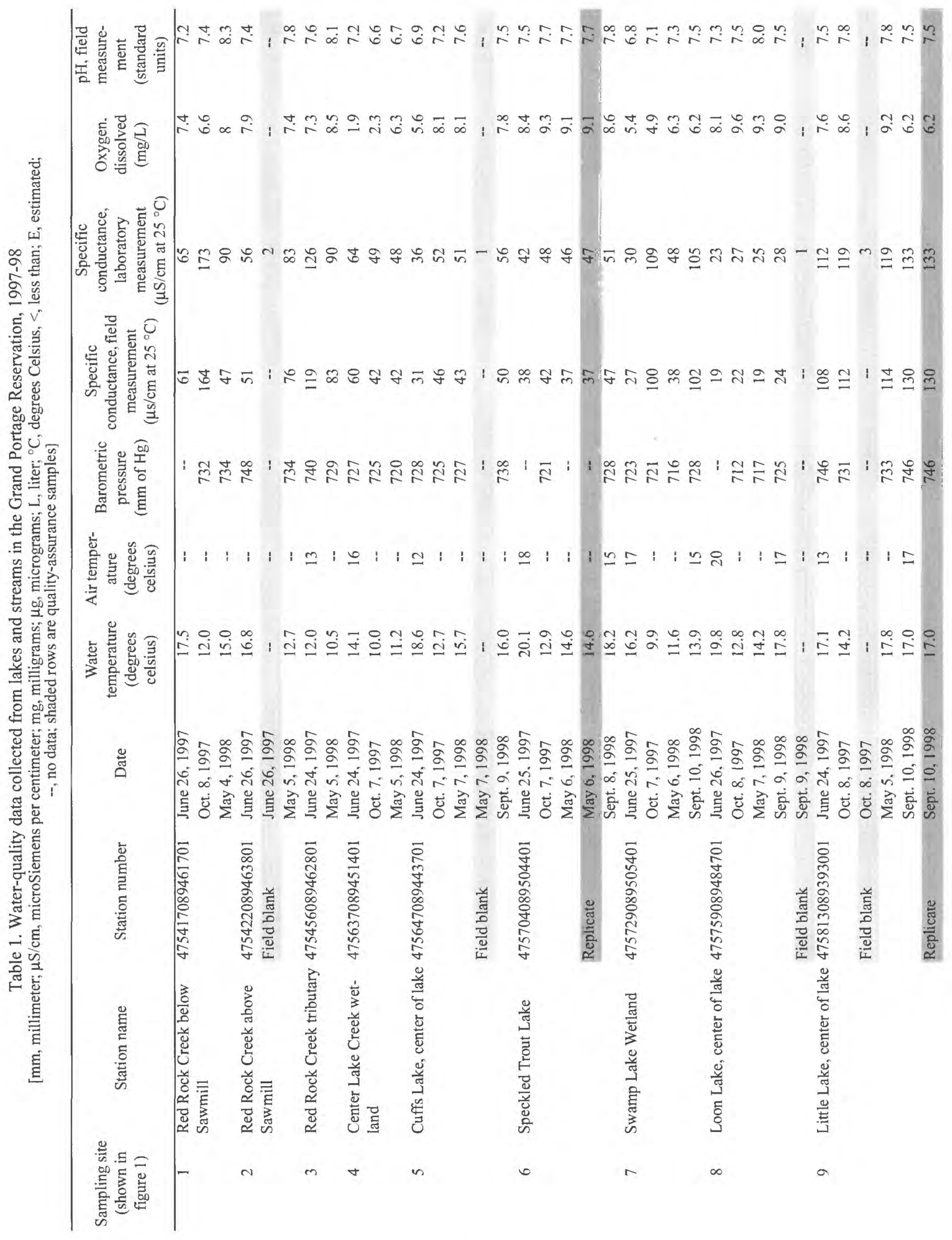




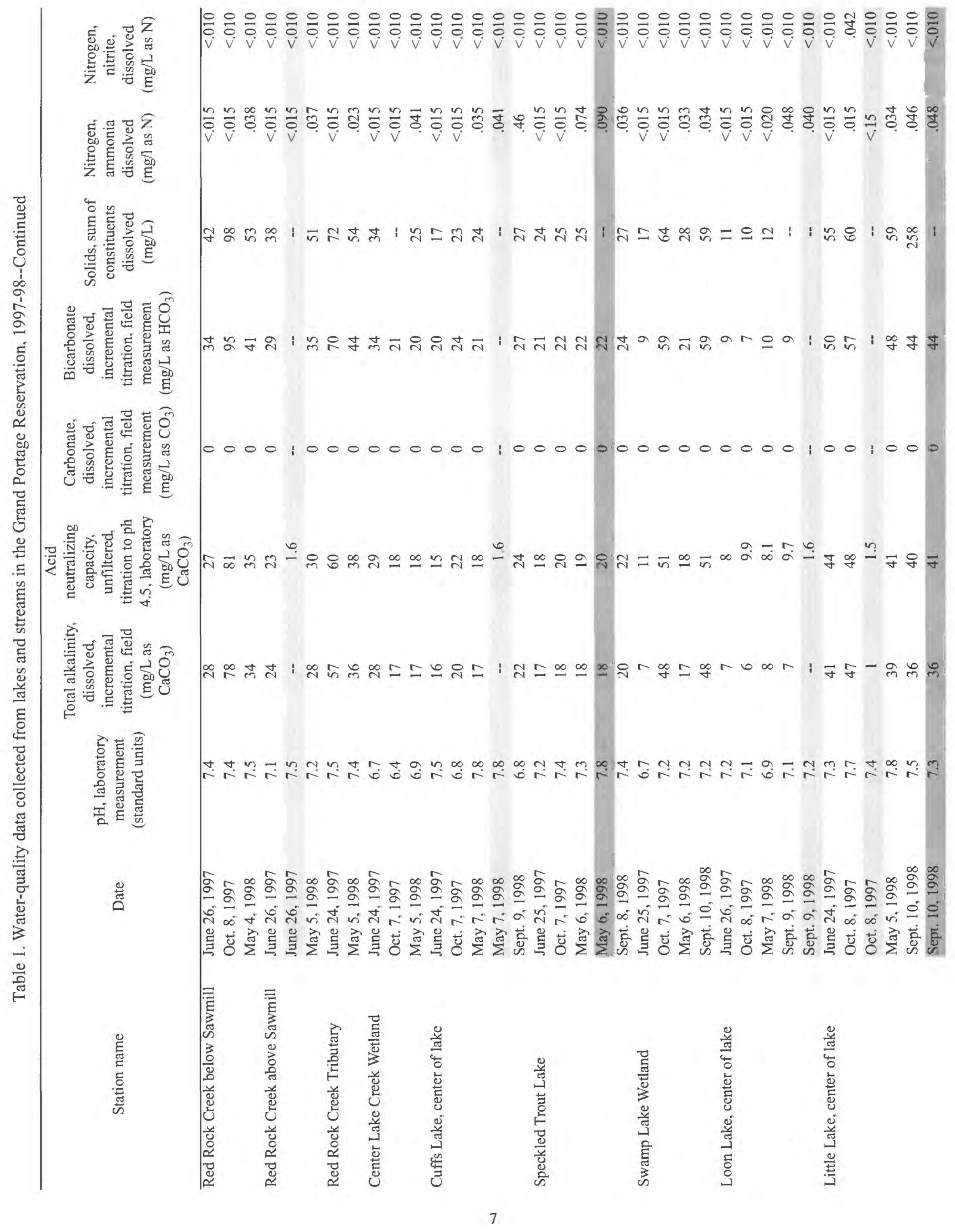




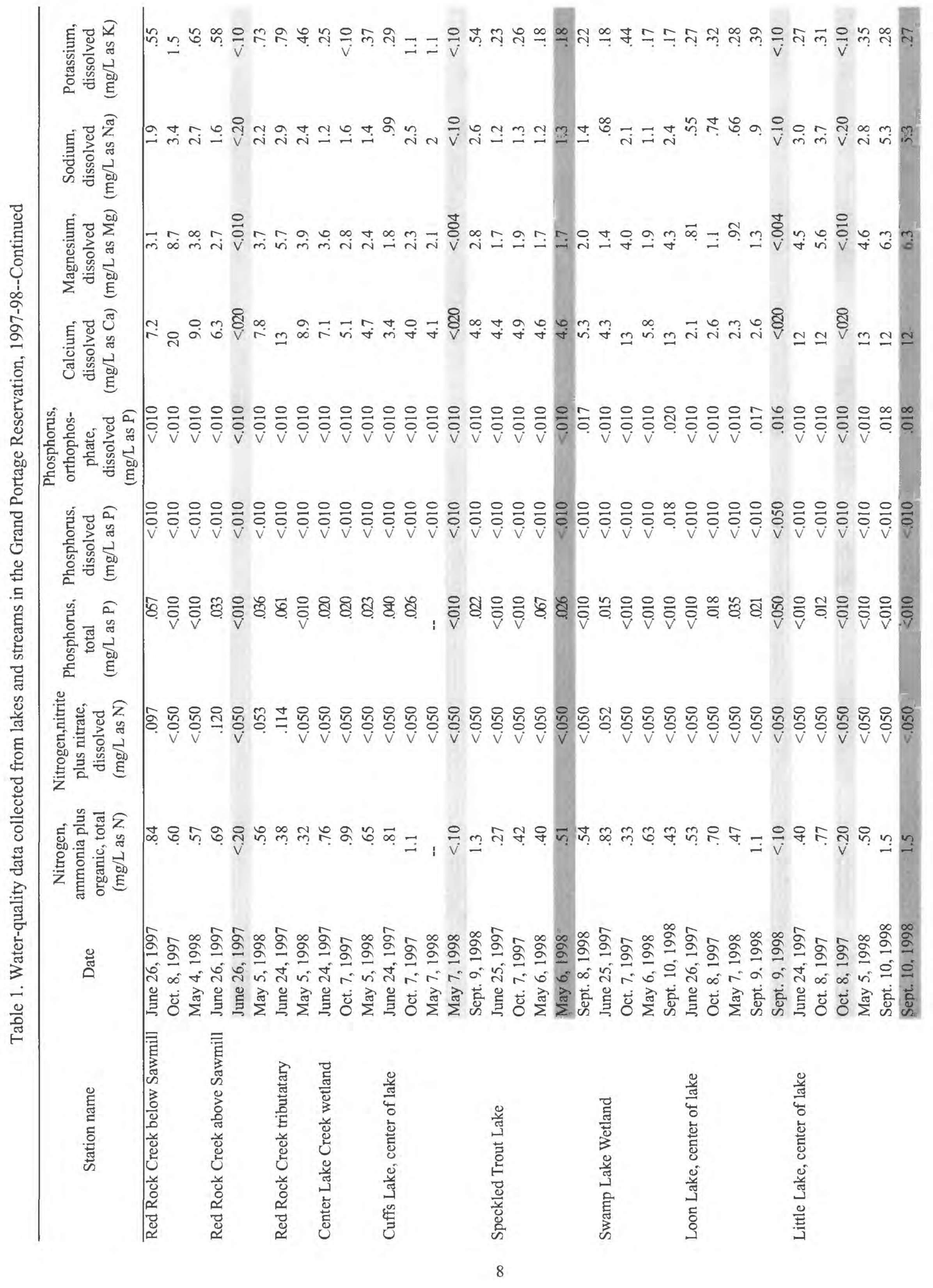




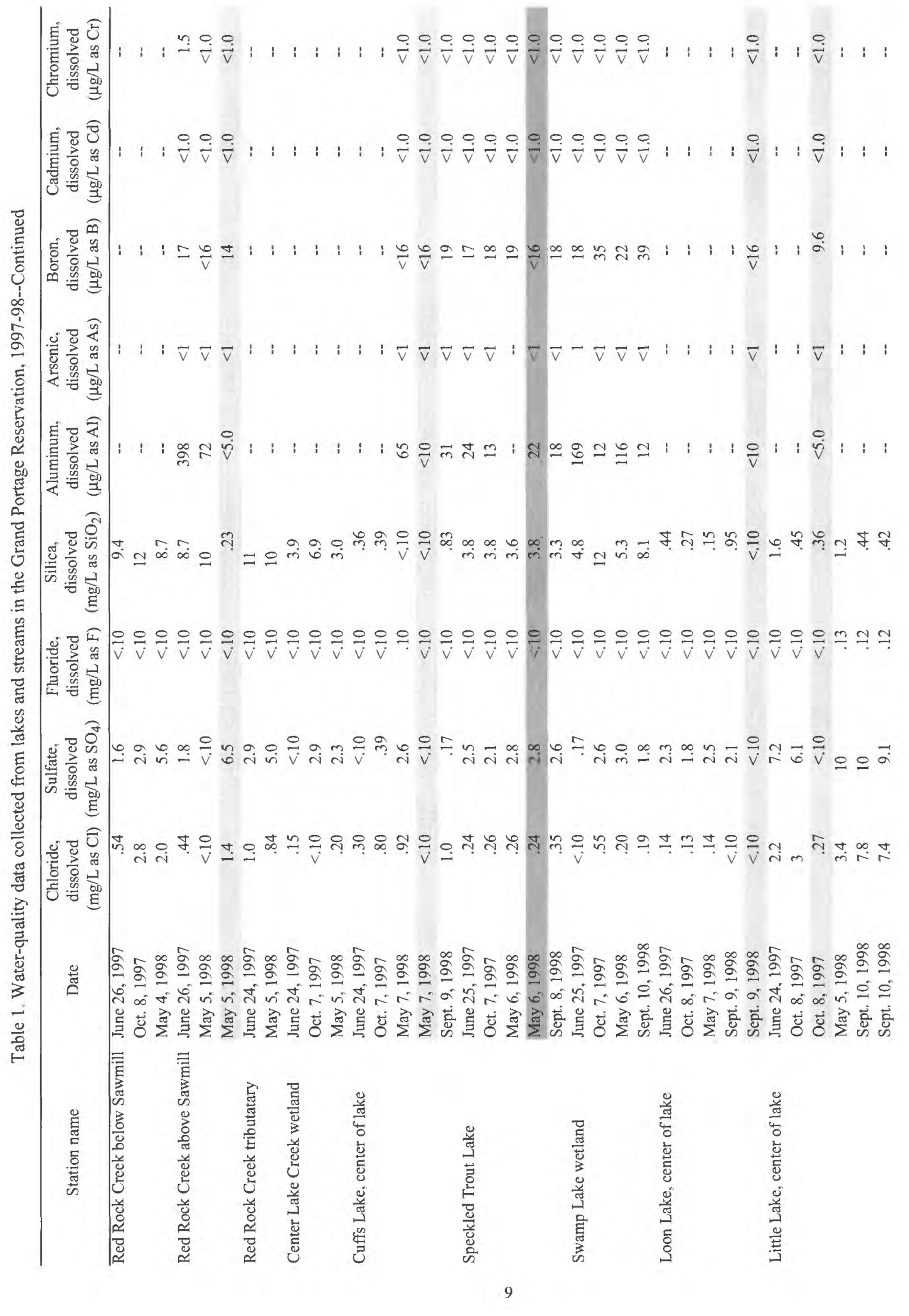




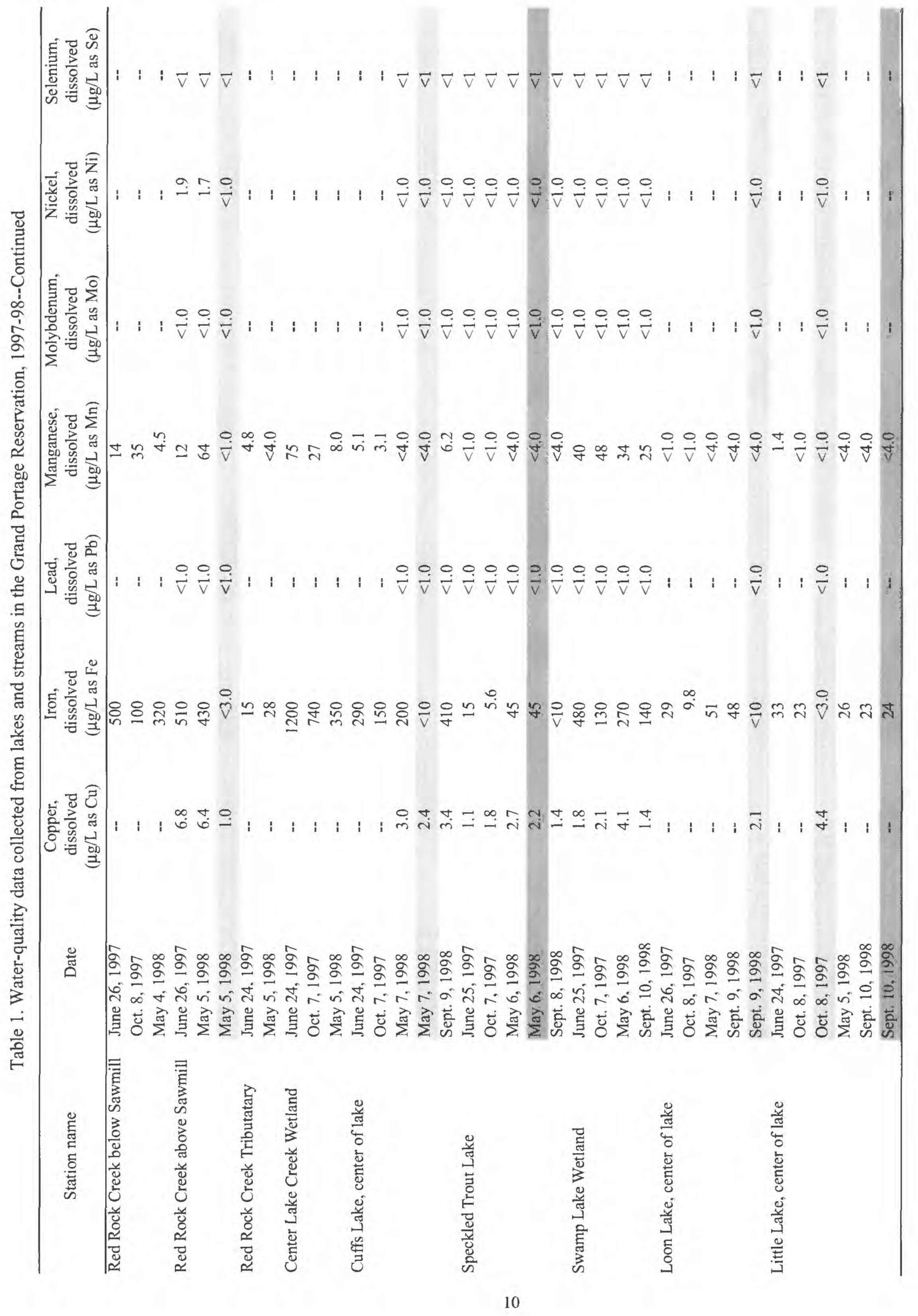


Table 2.. Physical Characteristics of Cuffs, Little, Loon, and Speckled Trout Lakes, Grand Portage Reservation, 1997--98

[The lakes were divided into 3 equal areas. The measurements were made at the center of each area. The Station column is the area that the measurements were made in: $\mathrm{C}$, center; $\mathrm{E}$, eastern; W, western; N. northern; S., southern; NE, northeastern; SW, southwestern. ${ }^{\circ} \mathrm{C}$, degrees celsius; $\mu \mathrm{S} / \mathrm{cm}$, microSiemens per centimeter at $25^{\circ} \mathrm{C} ; \mathrm{mg} / \mathrm{L}$, milligrams per liter; --, no data]

\begin{tabular}{|c|c|c|c|c|c|c|c|c|c|c|}
\hline Lake name & Date & Station & $\begin{array}{c}\text { Depth of } \\
\text { lake } \\
\text { (feet) }\end{array}$ & $\begin{array}{l}\text { Secchi disk } \\
\text { depth } \\
\text { (feet) }\end{array}$ & $\begin{array}{l}\text { Depth of } \\
\text { measurement } \\
\text { (feet) }\end{array}$ & $\mathrm{pH}$ & $\begin{array}{c}\text { Temperature } \\
\left({ }^{\circ} \mathrm{C}\right)\end{array}$ & $\begin{array}{c}\text { Specific } \\
\text { conductivity } \\
(\mu \mathrm{S} / \mathrm{cm})\end{array}$ & $\begin{array}{l}\text { Dissolved } \\
\text { oxygen } \\
(\mathrm{mg} / \mathrm{L})\end{array}$ & $\begin{array}{c}\text { Dissolved } \\
\text { oxygen } \\
\text { (percent } \\
\text { saturation) }\end{array}$ \\
\hline \multirow[t]{14}{*}{ Cuffs Lake } & $6 / 24 / 97$ & $\mathrm{NE}$ & 2.6 & $>2.6$ & 1.3 & 7.1 & $18 . \overline{9}$ & 32 & 5.5 & 57 \\
\hline & & $\mathrm{C}$ & 2.8 & $>2.8$ & 1.4 & 7.0 & 18.4 & 31 & 5.5 & 62 \\
\hline & & SW & 2.1 & $>2.1$ & 1.0 & 6.8 & 18.4 & 30 & 5.8 & 62 \\
\hline & $7 / 10 / 97$ & NE & 1.0 & -- & 0.5 & 7.3 & 12.8 & 45 & 8.0 & 79 \\
\hline & & $\mathrm{C}$ & .5 & -- & .02 & 7.1 & 12.6 & 45 & 8.2 & 80 \\
\hline & & SW & 1.0 & -- & .05 & 7.1 & 12.7 & 45 & 8.3 & 82 \\
\hline & $5 / 7 / 98$ & NE & 3.8 & $>3.8$ & 1 & 7.6 & 15.7 & 43 & 8.0 & 84 \\
\hline & & & & & 3.3 & 7.5 & 15.6 & 43 & 7.8 & 81 \\
\hline & & $\mathrm{C}$ & 4.0 & $>4.0$ & 1 & 7.5 & 15.8 & 43 & 8.1 & 84 \\
\hline & & & & & 3.3 & 7.2 & 15.6 & 39 & 6.8 & 70 \\
\hline & & SW & 3.5 & $>3.5$ & 1 & 7.6 & 16.0 & 43 & 8.4 & 87 \\
\hline & $9 / 9 / 98$ & NE & 2.5 & 2.4 & 1.25 & 7.9 & 16.4 & 51 & 7.1 & 74 \\
\hline & & $\mathrm{C}$ & 2.6 & $>2.6$ & 1.3 & 7.4 & 15.8 & 50 & 7.7 & 79 \\
\hline & & SW & 2.2 & $>2.2$ & 1.1 & 7.3 & 15.8 & 50 & 8.7 & 92 \\
\hline \multirow[t]{12}{*}{ Little Lake } & $6 / 24 / 97$ & $\mathrm{E}$ & 2.3 & -- & 1.2 & 7.7 & 17.2 & 109 & 7.3 & 78 \\
\hline & & $\mathrm{C}$ & 2.3 & - & 1.2 & 7.4 & 17.2 & 108 & 7.8 & 82 \\
\hline & & W & 2.0 & -- & 1.0 & 7.3 & 16.8 & 108 & 8.0 & 84 \\
\hline & $8 / 10 / 97$ & $\mathrm{E}$ & -- & -- & -- & 8.2 & 13.7 & 110 & 7.7 & 84 \\
\hline & & $\mathrm{C}$ & - & -- & -- & 7.6 & 14.4 & 111 & 9.0 & 90 \\
\hline & & W & - & -- & -- & 7.6 & 14.5 & 116 & 9.2 & 96 \\
\hline & $5 / 5 / 98$ & E & 2.2 & $>2.2$ & 1.1 & 8.0 & 17.8 & 114 & 9.2 & 100 \\
\hline & & $\mathrm{C}$ & 2.2 & $>2.2$ & 1.1 & 7.8 & 17.8 & 114 & 9.2 & 100 \\
\hline & & W & 2.5 & $>2.5$ & 1.2 & 7.6 & 17.8 & 114 & 9.2 & 100 \\
\hline & $9 / 10 / 98$ & E & $<1.0$ & -- & 0.5 & 7.6 & 16.7 & 126 & 6.7 & 68 \\
\hline & & $\mathrm{C}$ & $<1.0$ & - & 0.5 & 7.4 & 17.2 & 131 & 5.8 & 68 \\
\hline & & W & $<1.0$ & - & 0.5 & 7.4 & 17.4 & 134 & 6.0 & 64 \\
\hline \multirow[t]{18}{*}{ Loon Lake } & $6 / 26 / 98$ & SW & 4.7 & 3.9 & 1 & 7.7 & 19.8 & 19 & 8.1 & 94 \\
\hline & & & & & 2 & 7.6 & 19.8 & 19 & 8.1 & 94 \\
\hline & & & & & 3 & 7.5 & 19.8 & 19 & 8.1 & 94 \\
\hline & & & & & 4 & 7.5 & 19.7 & 19 & 8.2 & 94 \\
\hline & & $\mathrm{C}$ & 4.8 & 3.8 & 1 & 7.3 & 19.8 & 19 & 8.1 & 94 \\
\hline & & & & & 2 & 7.2 & 19.8 & 19 & 8.1 & 94 \\
\hline & & & & & 3 & 7.2 & 19.9 & 19 & 8.1 & 94 \\
\hline & & & & & 4 & 7.3 & 19.8 & 19 & 8.1 & 94 \\
\hline & & NE & 5.1 & 4.1 & 1 & 7.1 & 19.7 & 19 & 8.1 & 94 \\
\hline & & & & & 2 & 7.1 & 19.7 & 19 & 8.1 & 94 \\
\hline & & & & & 3 & 7.1 & 19.7 & 19 & 8.1 & 94 \\
\hline & & & & & 4 & 7.1 & 19.6 & 19 & 8.1 & 94 \\
\hline & & & & & 4.6 & 7.1 & 19.5 & 19 & 7.4 & - \\
\hline & $8 / 10 / 97$ & SW & 4.3 & 3.3 & 0.5 & 7.6 & 12.7 & 22 & 9.6 & 96 \\
\hline & & & & & 1.5 & 7.6 & 12.7 & 22 & 9.5 & 96 \\
\hline & & & & & 2.5 & 7.5 & 12.6 & 22 & 9.5 & 96 \\
\hline & & & & & 3.5 & 7.5 & 12.6 & 22 & 9.5 & 96 \\
\hline & & $\mathrm{C}$ & 4.0 & 3.3 & 0.5 & 7.4 & 12.8 & 22 & 9.7 & 98 \\
\hline
\end{tabular}


Table 2.. Physical Characteristics of Cuffs, Little, Loon, and Speckled Trout Lakes, Grand Portage Reservation, 1997--98

[The lakes were divided into 3 equal areas. The measurements were made at the center of each area. The Station column is the area that the measurements were made in: C, center; E, eastern; W, western; N, northern; S., southern; NE, northeastern; SW, southwestern. ${ }^{\circ} \mathrm{C}$, degrees celsius; $\mu \mathrm{S} / \mathrm{cm}$, microSiemens per centimeter at $25^{\circ} \mathrm{C} ; \mathrm{mg} / \mathrm{L}$, milligrams per liter; --, no data]

\begin{tabular}{|c|c|c|c|c|c|c|c|c|c|c|}
\hline Lake name & Date & Station & $\begin{array}{l}\text { Depth of } \\
\text { lake } \\
\text { (feet) }\end{array}$ & $\begin{array}{l}\text { Secchi disk } \\
\text { depth } \\
\text { (feet) }\end{array}$ & $\begin{array}{l}\text { Depth of } \\
\text { measurement } \\
\text { (feet) }\end{array}$ & $\mathrm{pH}$ & $\begin{array}{c}\text { Temperature } \\
\left({ }^{\circ} \mathrm{C}\right)\end{array}$ & $\begin{array}{c}\text { Specific } \\
\text { conductivity } \\
(\mu \mathrm{S} / \mathrm{cm})\end{array}$ & $\begin{array}{c}\text { Dissolved } \\
\text { oxygen } \\
(\mathrm{mg} / \mathrm{L})\end{array}$ & $\begin{array}{c}\text { Dissolved } \\
\text { oxygen } \\
\text { (percent } \\
\text { saturation) }\end{array}$ \\
\hline \multirow{25}{*}{$\begin{array}{l}\text { Loon Lake-- } \\
\text { Continuted }\end{array}$} & $8 / 10 / 97$ & & & & 1.5 & 7.5 & 12.8 & 22 & 9.7 & 98 \\
\hline & & & & & 2.5 & 7.4 & 12.8 & 22 & 9.7 & 98 \\
\hline & & & & & 3.5 & 7.4 & 12.8 & 22 & 9.7 & 98 \\
\hline & & NE & 4.5 & 3.7 & 0.5 & 7.4 & 13.0 & 22 & 9.7 & 98 \\
\hline & & & & & 1.5 & 7.4 & 12.9 & 22 & 9.7 & 98 \\
\hline & & & & & 2.5 & 7.4 & 13.0 & 22 & 9.7 & 98 \\
\hline & & & & & 3.5 & 7.4 & 12.9 & 22 & 9.7 & 98 \\
\hline & $5 / 7 / 98$ & SW & 4.2 & 4.0 & 1.0 & 8.1 & 14.2 & 18 & 9.0 & 90 \\
\hline & & & & & 1.6 & 8.0 & 14.2 & 18 & 9.0 & 90 \\
\hline & & & & & 3.3 & 8.0 & 14.2 & 18 & 9.1 & 91 \\
\hline & & C & 4.2 & 4.0 & 1.0 & 8.0 & 14.3 & 19 & 9.4 & 94 \\
\hline & & & & & 1.6 & 7.9 & 14.3 & 19 & 9.3 & 93 \\
\hline & & & & & 3.3 & 7.8 & 14.3 & 19 & 9.3 & 93 \\
\hline & & NE & 4.9 & 4.2 & 1.0 & 8.0 & 14.8 & 19 & 9.3 & 95 \\
\hline & & & & & 1.6 & 7.9 & 14.8 & 19 & 9.3 & 94 \\
\hline & & & & & 3.3 & 7.8 & - & 19 & 9.3 & 95 \\
\hline & 9/9/98 & SW & 3.4 & 3.2 & 1.0 & 7.7 & 17.5 & 24 & 9.0 & 98 \\
\hline & & & & & 2.0 & 7.8 & 17.5 & 24 & 9.0 & 97 \\
\hline & & & & & 3.0 & 7.8 & 17.5 & 24 & 9.0 & 97 \\
\hline & & $\mathrm{C}$ & 3.5 & 3.2 & 1.0 & 73 & 17.9 & 24 & 9.0 & 98 \\
\hline & & & & & 2.0 & 7.4 & 17.8 & 24 & 9.0 & 98 \\
\hline & & & & & 3.0 & 7.4 & 17.8 & 24 & 9.1 & 99 \\
\hline & & $\mathrm{NE}$ & 3.8 & 3.2 & 1.5 & 7.2 & 17.9 & 24 & 9.0 & 98 \\
\hline & & & & & 2.5 & 7.2 & 17.9 & 24 & 9.0 & 98 \\
\hline & & & & & 3.5 & 7.3 & 17.9 & 24 & 9.1 & 99 \\
\hline \multirow[t]{18}{*}{$\begin{array}{l}\text { Speckled Trout } \\
\text { Lake }\end{array}$} & $6 / 25 / 97$ & $\mathrm{NE}$ & 8.3 & 6.9 & 1.0 & 8.0 & 20.2 & 38 & 8.4 & 95 \\
\hline & & & & & 2.0 & 7.9 & 20.2 & 38 & 8.4 & 95 \\
\hline & & & & & 3.0 & 7.9 & 20.2 & 38 & 8.4 & 95 \\
\hline & & & & & 4.0 & 7.8 & 20.1 & 38 & 8.4 & 95 \\
\hline & & & & & 5.0 & 7.8 & 20.2 & 38 & 8.5 & 96 \\
\hline & & & & & 6.0 & 7.8 & 20.1 & 38 & 8.5 & 96 \\
\hline & & & & & 7.0 & 7.7 & 20.1 & 38 & 8.5 & 96 \\
\hline & & & & & 7.8 & 7.6 & 20.1 & 38 & 7.7 & 90 \\
\hline & & $\mathrm{C}$ & -- & 6.2 & 1.0 & 7.5 & 20.2 & 39 & 8.4 & 95 \\
\hline & & & & & 2.0 & 7.5 & 20.2 & 39 & 8.4 & 95 \\
\hline & & & & & 3.0 & 7.5 & 20.2 & 38 & 8.4 & 95 \\
\hline & & & & & 4.0 & 7.5 & 20.2 & 38 & 8.4 & 95 \\
\hline & & & & & 5.0 & 7.5 & 20.1 & 38 & 8.4 & 95 \\
\hline & & & & & 6.0 & 7.5 & 20.1 & 38 & 8.5 & 95 \\
\hline & & & & & 7.0 & 7.5 & 20.0 & 38 & 8.5 & 96 \\
\hline & & & & & 8.0 & 7.5 & 19.8 & 38 & 8.6 & 96 \\
\hline & & & & & 9.0 & 7.5 & 19.8 & 38 & 8.6 & 96 \\
\hline & & & & & 9.7 & 7.5 & 19.8 & 38 & 8.3 & -- \\
\hline
\end{tabular}


Table 2.. Physical Characteristics of Cuffs, Little. Loon, and Speckled Trout Lakes, Grand Portage Reservation, 1997--98

[The lakes were divided into 3 equal areas. The measurements were made at the center of each area. The Station column is the area that the measurements were made in: $\mathrm{C}$, center; E, eastern; W, western; N, northern; S., southern; NE, northeastern; SW, southwestern. ${ }^{\circ} \mathrm{C}$, degrees celsius; $\mu \mathrm{S} / \mathrm{cm}$, microSiemens per centimeter at $25^{\circ} \mathrm{C} ; \mathrm{mg} / \mathrm{L}$, milligrams per liter; --, no data]

\begin{tabular}{|c|c|c|c|c|c|c|c|c|c|c|}
\hline Lake name & Date & Station & $\begin{array}{l}\text { Depth of } \\
\text { lake } \\
\text { (feet) }\end{array}$ & $\begin{array}{c}\text { Secchi disk } \\
\text { depth } \\
\text { (feet) }\end{array}$ & $\begin{array}{c}\text { Depth of } \\
\text { measurement } \\
\text { (feet) }\end{array}$ & $\mathrm{pH}$ & $\begin{array}{c}\text { Temperature } \\
\left({ }^{\circ} \mathrm{C}\right)\end{array}$ & $\begin{array}{c}\text { Specific } \\
\text { conductivity } \\
(\mu \mathrm{S} / \mathrm{cm})\end{array}$ & $\begin{array}{c}\text { Dissolved } \\
\text { oxygen } \\
(\mathrm{mg} / \mathrm{L})\end{array}$ & $\begin{array}{c}\text { Dissolved } \\
\text { oxygen } \\
\text { (percent } \\
\text { saturation) }\end{array}$ \\
\hline \multirow[t]{43}{*}{$\begin{array}{l}\text { Speckled Trout } \\
\text { Lake--Continued }\end{array}$} & $6 / 25 / 97$ & SW & 4.9 & $>4.9$ & 1.0 & 7.4 & 20.2 & 38 & 8.4 & 94 \\
\hline & & & & & 2.0 & 7.4 & 20.1 & 38 & 8.4 & 94 \\
\hline & & & & & 3.0 & 7.4 & 20.1 & 39 & 8.3 & 94 \\
\hline & & & & & 4.0 & 7.4 & 20.0 & 39 & 8.2 & 92 \\
\hline & & & & & 4.6 & 7.4 & 20.0 & 39 & 7.8 & 84 \\
\hline & $7 / 10 / 97$ & $\mathrm{NE}$ & 7.5 & 5.4 & .5 & 7.9 & 13.1 & 42 & 9.2 & 92 \\
\hline & & & & & 1.5 & 7.9 & 13.1 & 42 & 9.2 & 92 \\
\hline & & & & & 2.5 & 7.8 & 13.1 & 42 & 9.2 & 92 \\
\hline & & & & & 3.5 & 7.8 & 13.1 & 42 & 9.2 & 92 \\
\hline & & & & & 4.5 & 7.8 & 13.0 & 42 & 9.3 & 93 \\
\hline & & & & & 5.5 & 7.8 & 12.9 & 42 & 9.3 & 93 \\
\hline & & & & & 6.5 & 7.8 & 12.8 & 41 & 9.3 & 93 \\
\hline & & & & & 7.0 & 7.8 & 12.8 & 42 & 7.1 & 76 \\
\hline & & C & 8.7 & 5.3 & .5 & 7.7 & 12.9 & 42 & 9.2 & 93 \\
\hline & & & & & 1.5 & 7.7 & 12.9 & 42 & 9.2 & 93 \\
\hline & & & & & 2.5 & 7.7 & 12.9 & 42 & 9.2 & 93 \\
\hline & & & & & 3.5 & 7.7 & 12.9 & 41 & 9.2 & 93 \\
\hline & & & & & 4.5 & 7.7 & 12.8 & 42 & 9.2 & 93 \\
\hline & & & & & 5.5 & 7.7 & 12.8 & 42 & 9.3 & 93 \\
\hline & & & & & 6.5 & 7.7 & 12.7 & 41 & 9.3 & 93 \\
\hline & & & & & 7.5 & 7.7 & 12.7 & 41 & 9.3 & 93 \\
\hline & & & & & 8.5 & 7.7 & 12.7 & 42 & 9.3 & 78 \\
\hline & & SW & 5.4 & 5.4 & .5 & 7.6 & 12.9 & 41 & 9.2 & 93 \\
\hline & & & & & 1.5 & 7.6 & 12.9 & 42 & 9.3 & 93 \\
\hline & & & & & 2.5 & 7.6 & 12.9 & 41 & 9.3 & 93 \\
\hline & & & & & 3.5 & 7.7 & 12.9 & 41 & 9.3 & 94 \\
\hline & & & & & 4.5 & 7.7 & 12.9 & 41 & 9.4 & 94 \\
\hline & & & & & 5.0 & 7.4 & 12.9 & 43 & 9.3 & 62 \\
\hline & $5 / 6 / 98$ & $\mathrm{NE}$ & 7.9 & 6.4 & 1.0 & 8.0 & 14.6 & 36 & 9.1 & 94 \\
\hline & & & & & 1.6 & 7.9 & 14.6 & 37 & 9.2 & 94 \\
\hline & & & & & 3.3 & 7.9 & 14.5 & 37 & 9.2 & 94 \\
\hline & & & & & 4.9 & 7.8 & 14.4 & 37 & 9.1 & 94 \\
\hline & & & & & 6.6 & 7.8 & 14.1 & 36 & 9.2 & 93 \\
\hline & & $\mathrm{C}$ & 12.5 & - & 1.6 & 7.8 & 14.7 & 37 & 9.1 & 94 \\
\hline & & & & & 3.3 & 7.7 & 14.3 & 37 & 9.1 & 94 \\
\hline & & & & & 4.9 & 7.7 & 14.2 & 37 & 9.1 & 94 \\
\hline & & & & & 6.6 & 7.7 & 14.1 & 37 & 9.2 & 94 \\
\hline & & & & & 8.2 & 7.7 & 14.1 & 37 & 9.2 & 94 \\
\hline & & & & & 9.8 & 7.6 & 14.0 & 37 & 9.3 & 95 \\
\hline & & & & & 11.5 & 72 & 13.8 & 37 & 8.7 & 87 \\
\hline & & SW & 3.6 & - & 1.6 & 7.7 & 14.9 & 37 & 9.3 & 97 \\
\hline & & & & & 3.3 & 7.8 & 14.6 & 39 & 9.2 & 94 \\
\hline & $9 / 9 / 98$ & NE & 9.9 & 5.5 & 1.5 & 7.8 & 18.5 & 47 & 8.5 & 92 \\
\hline
\end{tabular}


Table 2.. Physical Characteristics of Cuffs, Little, Loon, and Speckled Trout Lakes, Grand Portage Reservation, 1997--98 [The lakes were divided into 3 equal areas. The measurements were made at the center of each area. The Station column is the area that the measurements were made in: C, center; E, eastern; W, western; N, northern; S., southern; NE, northeastern; SW, southwestern. ${ }^{\circ} \mathrm{C}$, degrees celsius; $\mu \mathrm{S} / \mathrm{cm}$, microSiemens per centimeter at $25^{\circ} \mathrm{C} ; \mathrm{mg} / \mathrm{L}$, milligrams per liter; --, no data]

\begin{tabular}{|c|c|c|c|c|c|c|c|c|c|c|}
\hline Lake name & Date & Station & $\begin{array}{l}\text { Depth of } \\
\text { lake } \\
\text { (feet) }\end{array}$ & $\begin{array}{l}\text { Secchi disk } \\
\text { depth } \\
\text { (feet) }\end{array}$ & $\begin{array}{c}\text { Depth of } \\
\text { measurement } \\
\text { (feet) }\end{array}$ & $\mathrm{pH}$ & $\begin{array}{c}\text { Temperature } \\
\left({ }^{\circ} \mathrm{C}\right)\end{array}$ & $\begin{array}{c}\text { Specific } \\
\text { conductivity } \\
(\mu \mathrm{S} / \mathrm{cm})\end{array}$ & $\begin{array}{c}\text { Dissolved } \\
\text { oxygen } \\
(\mathrm{mg} / \mathrm{L})\end{array}$ & $\begin{array}{l}\text { Dissolved } \\
\text { oxygen } \\
\text { (percent } \\
\text { saturation) }\end{array}$ \\
\hline \multirow[t]{20}{*}{$\begin{array}{l}\text { Speckled Trout } \\
\text { Lake--Continued }\end{array}$} & $9 / 9 / 98$ & & & & 2.5 & 7.8 & 18.5 & 47 & 8.5 & 92 \\
\hline & & & & & 3.5 & 7.8 & 18.4 & 47 & 8.5 & 92 \\
\hline & & & & & 4.5 & 7.8 & 18.3 & 47 & 8.5 & 92 \\
\hline & & & & & 5.5 & 7.8 & 18.2 & 47 & 8.6 & 92 \\
\hline & & & & & 6.5 & 7.8 & 18.1 & 46 & 8.6 & 93 \\
\hline & & & & & 7.5 & 7.6 & 18.1 & 47 & 8.7 & 92 \\
\hline & & & & & 8.4 & 7.1 & 18.4 & 60 & 0.2 & -- \\
\hline & & $\mathrm{C}$ & 9.9 & 5.5 & 1.5 & 7.8 & 18.4 & 47 & 8.5 & 92 \\
\hline & & & & & 2.5 & 7.8 & 18.4 & 47 & 8.5 & 92 \\
\hline & & & & & 3.5 & 7.8 & 18.4 & 47 & 8.5 & 92 \\
\hline & & & & & 4.5 & 7.8 & 18.2 & 47 & 8.5 & 92 \\
\hline & & & & & 5.5 & 7.8 & 18.1 & 47 & 8.5 & 92 \\
\hline & & & & & 6.5 & 7.9 & 18.5 & 47 & 8.5 & 92 \\
\hline & & & & & 7.5 & 7.9 & 17.9 & 47 & 8.6 & 92 \\
\hline & & & & & 8.5 & 7.8 & 17.8 & 47 & 8.6 & 92 \\
\hline & & & & & 9.5 & 7.8 & 17.7 & 47 & 8.9 & 93 \\
\hline & & SW & 5.0 & 5.5 & 1.5 & 79 & 18.2 & 47 & 8.6 & 93 \\
\hline & & & & & 2.5 & 7.8 & 18.2 & 47 & 8.6 & 93 \\
\hline & & & & & 3.5 & 7.9 & 18.2 & 47 & 8.6 & 93 \\
\hline & & & & & 4.5 & 7.8 & 18.2 & 47 & 8.7 & 93 \\
\hline
\end{tabular}


Table 3. Relative percent difference between concentrations of constituents in the sample and replicate sample from Speckled Trout Lake and Little Lake

$\left[\mu \mathrm{S} / \mathrm{cm}\right.$ at $25^{\circ} \mathrm{C}$, microSiemens per centimeter at 25 degrees Celsius; $\mathrm{mg} / \mathrm{L}$, milligrams per liter; $\mu \mathrm{g} / \mathrm{L}$, micrograms per liter; $<$, less than; --, relative percent difference not calculated]

\begin{tabular}{|c|c|c|c|c|c|c|}
\hline & \multicolumn{3}{|c|}{$\begin{array}{c}\text { Speckled Trout Lake, } \\
\text { Station } 475704089504401, \\
\text { May } 6,1998\end{array}$} & \multicolumn{3}{|c|}{$\begin{array}{l}\text { Little Lake, center of lake, } \\
\text { Station } 475813089393001 \text {, } \\
\text { September 10, } 1998\end{array}$} \\
\hline & Sample & Replicate & $\begin{array}{l}\text { Relative } \\
\text { percent } \\
\text { difference }\end{array}$ & Sample & Replicate & $\begin{array}{l}\text { Relative } \\
\text { percent } \\
\text { difference }\end{array}$ \\
\hline $\begin{array}{l}\text { Specific conductance, laboratory measurement, } \\
\left(\mu \mathrm{S} / \mathrm{cm} \text { at } 25^{\circ} \mathrm{C}\right)\end{array}$ & 46 & 47 & -1.1 & 133 & 133 & 0 \\
\hline pH, laboratory measurement (standard units) & 7.3 & 7.8 & -3.3 & 7.5 & 7.3 & 1.4 \\
\hline $\begin{array}{l}\text { Acid neutralizing capacity, unfiltered, titration To pH } 4.5 \text {, } \\
\text { laboratory, }\left(\mathrm{mg} / \mathrm{L} \text { as } \mathrm{CaCO}_{3}\right)\end{array}$ & 19 & 20 & -2.6 & 40 & 41 & -1.2 \\
\hline Nitrogen, ammonia dissolved (mg/L as $\mathrm{N}$ ) & 0.074 & 0.090 & -9.8 & 0.046 & $0.04^{\circ}$ & -2.1 \\
\hline $\begin{array}{l}\text { Nitrogen, Nitrite, } \\
\text { Dissolved (mg/L as N) }\end{array}$ & $<.010$ & $<.010$ & -- & $<.010$ & $<.010$ & -- \\
\hline Nitrogen, ammonia plus organic, total ( $\mathrm{mg} / \mathrm{L}$ as $\mathrm{N}$ ) & 0.40 & 0.51 & -12.1 & 1.5 & 1.5 & 0 \\
\hline Nitrogen, nitrite plus nitrate. dissolved ( $\mathrm{mg} / \mathrm{L}$ as $\mathrm{N})$ & $<.050$ & $<.050$ & -- & $<.050$ & $<.050$ & -- \\
\hline Phosphorus, total (mg/L as P) & 0.067 & 0.026 & 44.1 & $<.010$ & $<010$ & $\rightarrow$ \\
\hline Phosphorus, dissolved (mg/L as P) & $<.010$ & $<.010$ & -- & $<.010$ & $<.010$ & - \\
\hline Phosphorus, orthophosphate, dissolved ( $\mathrm{mg} / \mathrm{L}$ as P) & $\$ .010$ & $<.010$ & - & 0.018 & 0.018 & 0 \\
\hline Calcium, dissolved (mg/L as $\mathrm{Ca})$ & 4.6 & 4.6 & 0 & 12 & 12 & 0 \\
\hline Magnesium, dissolved (mg/L as $\mathrm{Mg}$ ) & 1.7 & 1.7 & 0 & 6.3 & 6.3 & 0 \\
\hline Sodium, dissolved (mg/L as Na) & 1.2 & 1.3 & -4 & 5.3 & 5.3 & u \\
\hline Potassium, dissolved (mg/L as $\mathrm{K}$ ) & 0.18 & 0.18 & 0 & 0.28 & 0.27 & 1.8 \\
\hline Chluride, dissolved (mg/L as $\mathrm{Cl}$ ) & 0.26 & 0.24 & 4 & 7.8 & 7.4 & 2.6 \\
\hline Sulfate, dissolved ( $\mathrm{mg} / \mathrm{L}$ as $\mathrm{SO}_{4}$ ) & 2.8 & 2.8 & 0 & 10 & 9.1 & 4.7 \\
\hline Fluoride, dissolved (mg/L as F) & $<.10$ & $<10$ & - & 0.12 & 0.12 & 0 \\
\hline Silica, dissolved ( $\mathrm{mg} / \mathrm{L}$ as $\mathrm{SiO}_{2}$ ) & 3.6 & 3.8 & -2.7 & 0.44 & 0.42 & 2.3 \\
\hline Aluminum dissolved ( $\mu \mathrm{g} / \mathrm{L}$ as $\mathrm{Al})$ & -- & 22 & - & -- & - & -- \\
\hline Arsenic dissolved ( $\mu \mathrm{g} / \mathrm{L}$ as As) & - & $<1$ & - & - & - & - \\
\hline Boron dissolved $(\mu \mathrm{g} / \mathrm{L}$ as $\mathrm{B})$ & 19 & $<16$ & -- & -- & -- & - \\
\hline Cadmium dissolved $(\mu \mathrm{g} / \mathrm{L}$ as $\mathrm{Cd})$ & $<1.0$ & $<1.0$ & - & - & - & - \\
\hline Chromium dissulved ( $\mu \mathrm{g} / \mathrm{L}$ as $\mathrm{Cr}$ ) & $<1.0$ & $<1.0$ & -- & - & -- & -- \\
\hline Copper dissolved $(\mu \mathrm{g} / \mathrm{L}$ as $\mathrm{Cu})$ & 2.7 & 2.2 & 10.2 & - & - & -1 \\
\hline Iron, dissolved ( $\mu \mathrm{g} / \mathrm{L}$ as $\mathrm{Fe})$ & 45 & 45 & 0 & 23 & 24 & -2.1 \\
\hline Lead dissolved $(\mu \mathrm{g} / \mathrm{L}$ as $\mathrm{Pb})$ & $<1,0$ & $<1.0$ & - & - & - & -- \\
\hline Manganese dissolved ( $\mu \mathrm{g} / \mathrm{Ll}$ as $\mathrm{Mn})$ & $<4.0$ & $<4.0$ & -- & $<4.0$ & $<4.0$ & -- \\
\hline Molybdenum dissolved ( $\mu \mathrm{g} / \mathrm{L}$ as $\mathrm{Mo}$ ) & $<1.0$ & $<1.0$ & - & - & - & 9 \\
\hline Nickel dissolved $(\mu \mathrm{g} / \mathrm{Ll}$ as $\mathrm{Ni})$ & $<1.0$ & $<1.0$ & -- & -- & - & -- \\
\hline Selenium dissolved $(\mu \mathrm{g} / \mathrm{L}$ as $\mathrm{Se})$ & $<1$ & $<1$ & - & - & - & - \\
\hline
\end{tabular}




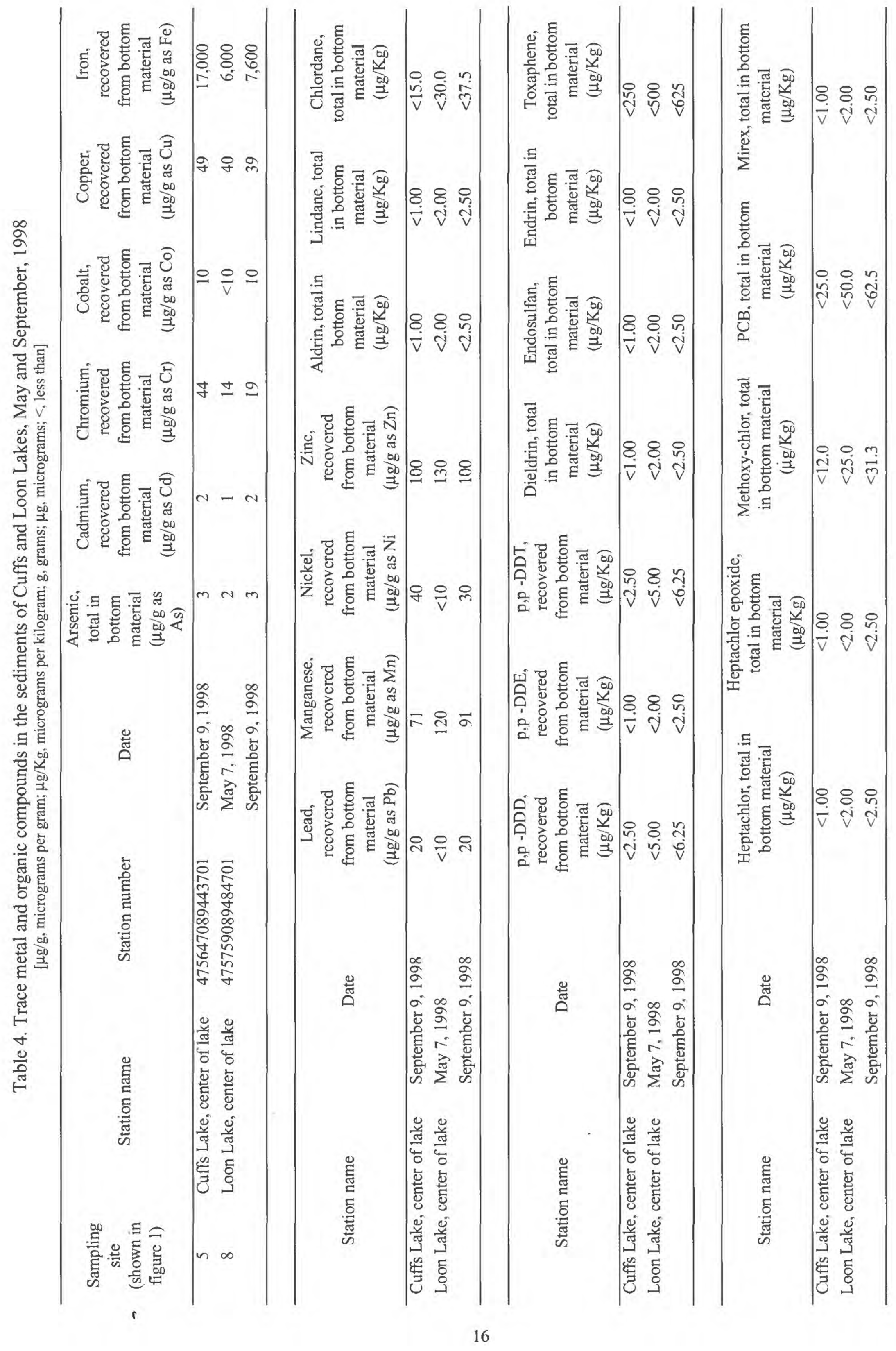

